\title{
ACTION COMPARÉE DE L'ÉRY'THROMYCINE ET DE L'AURÉOMYCINE SUR LA CROISSANCE DU POUSSIN ET STOCKAGE DE L'ÉRYTHROMYCINE DANS LES TISSUS
}

\author{
PAR \\ C. CALET \\ Station de Recherches avicoles, C. N. R. Z., Jouy-en-Josas (S. et O.).
}

\section{SOMMAIRE}

Mieux que l'auréomycine, l'érythromycine favorise le développement pondéral du Poussin et améliore le taux de conversion alimentaire. Son action positive sur la croissance est indépendante du mode d'élevage de l'animal. L'importance du transfert de l'érythromycine alimentaire au muscle et au foie est faible.

L'érythromycine est un nouvel antibiotique utilisé en médecine humaine dans le traitement des maladies infectieuses (HUNTER, I950). L,es qualités de cette substance, qui la font particulièrement bien apprécier, se manifestent par son spectre antibactérien très étendu et par la tolérance très marquée qu'elle présente vis-à-vis des différents tissus.

Ies essais d'emploi de l'érythromycine comme facteur de croissance sont rares. On peut citer les travaux de RERAT et al., I957 a-1957 $b$ sur le Rat qui démontrent la faculté de cet antibiotique d'accroittre le taux de croissance des animatx et d'améliorer l'indice de consommation.

Michei, et Françors, I955 ont démontré qu'il existe une relation entre l'aptitude d'un antibiotique à stimuler la croissance et son pouvoir d'inhiber les désaminases microbiennes. Or ces mêmes auteurs ont pu montrer que l'érythromycine exerce une action inhibitrice très importante vis-à-vis des désaminases microbiennes (Mrchel, M., I958).

Il est donc dès lors intéressant de mettre en évidence l'efficacité éventuelle de cet antibiotique ajouté à la ration pour la croissance du Poulet. 
Pour mieux mettre en valeur nos résultats, nous avons comparé l'efficacité pour la croissance de ce nouvel antibiotique, obtenu à l'état pur, à celui d'un autre antibiotique, le chlorhydrate d'auréomycine.

Tous nos résultats sont rapportés à ceux d'un lot témoin qui reçoit le régime de base seul.

\section{MATERIEL E'T MÉTHODES}

I38 poussins d'un jour, mâles, de race Rhode Wyandotte, sont répartis en 6 lots homogènes et identiques. Pour chacun des 3 régimes considérés un lot est élevé au sol, l'autre sur grillage.

Nous avons donc le dispositif d'élevage suivant:

\begin{tabular}{c|c|c|c}
\hline Nature du régime & Situıtion & Nombre de poussins & Lots \\
\hline Régime de base $\ldots \ldots \ldots$ & sur grillage & 23 & I \\
aúgime de base + auréo- & sur grillage & 23 & II \\
mycine ........... & au sol & 23 & III \\
Régime de base + éry- & sur grillage & 23 & IV \\
thromycine ......... & au sol & 23 & V \\
\hline
\end{tabular}

Dans tous les cas, la surface mise à la disposition des poussins d'un lot est la même. En dehors des variations portant sur les régimes et sur la nature de la cage, les poussins sont soumis aux mêmes soins d'élevage Ces régimes sont ainsi constitués:

a) Le régime de base :

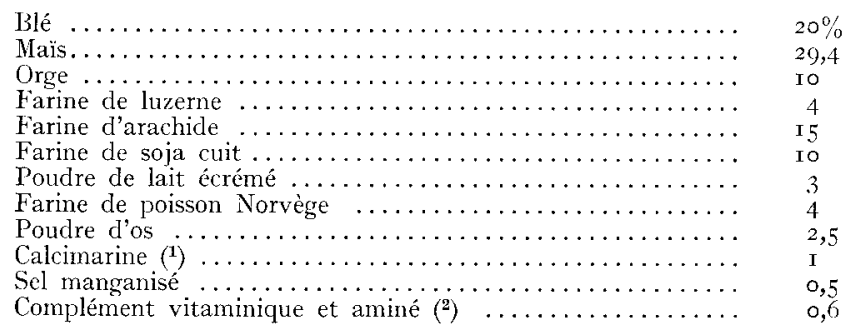

Ce régime de base titre $20,5 \mathrm{p}$. Ioo de matières azotées totales dont les protéines sont équilibrées en acides aminés.

b) Les régimes expérimentaux sont les suivants:

- Les lots témoins (I et II) reçoivent le régime de base seul.

(1) Complexe salin constitué de carbonate de chaux, carbonate de magnésie, phosphate tricalcique, chlorure de sodium.

(2) Le complément vitaminique et aminé assure la couverture des besoins vitaminiques.

Il permet de plus un équilibre satisfaisant des acides aminés de l'ensemble du régime. 
- Les lots "Auréomycine " (III et IV) reçoivent le régime de base enrichi de $20 \mathrm{mg}$ de chlorhydrate d'auréomycine par $\mathrm{kg}$ de ration.

- Les lots "Errythromycine " (V et VI) reçoivent le régime de base auquel on ajoute $20 \mathrm{mg} / \mathrm{kg}$ de ration d'Érythromycine de base (érythromycine pure).

Les animaux sont pesés individuellement chaque semaine et la consommation de nourriture est contrôlée chaque jour.

Les observations sur animal portent sur leur aspect, leur gain de poids, leur consommation de nourriture, et le stockage de l'érythromycine dans les tissus.

\section{TABLEAU I}

Moyenne des gains de poids et consommation d'aliments sous l'effet de l'auréomycine et de l'érythromycine.

\begin{tabular}{|c|c|c|c|c|c|c|}
\hline & \multicolumn{2}{|c|}{ Témoins } & \multicolumn{2}{|c|}{ Auréonycine } & \multicolumn{2}{|c|}{ Erythromycine } \\
\hline & Lot I & Lot II & Lot III & Lot IV & Lot V & Lot VI \\
\hline $\begin{array}{l}\text { Gain de poids à } 4 \text { semai- } \\
\text { nes }(g) \ldots \ldots \ldots \ldots \ldots \ldots \\
\text { Nourriture ingérée pen- } \\
\text { dant } 4 \text { semaines }(g) \ldots . \\
\text { Indice de consommation. }\end{array}$ & $\begin{array}{r}367 \\
\text { I } 608 \\
4,3^{8}\end{array}$ & $\begin{array}{c}373 \\
\text { I } 536 \\
4,11\end{array}$ & $\begin{array}{c}390 \\
\text { I } 525 \\
3,9 \mathrm{I}\end{array}$ & $\begin{array}{c}404 \\
\text { I } 463 \\
3,62\end{array}$ & $\begin{array}{r}433 \\
\text { I } 528 \\
3,66\end{array}$ & $\begin{array}{r}435 \\
\text { I } 490 \\
3,42\end{array}$ \\
\hline $\begin{array}{l}\text { Gain de poids â } 9 \text { semai- } \\
\text { nes }(g) \ldots \ldots \ldots \ldots \ldots . . . . . . \\
\text { Nourriture ingérée pen- } \\
\text { dant } 9 \text { semaines }(g) . . . \\
\text { Indice de consommation. }\end{array}$ & $\begin{array}{c}\text { I I I } 5 \\
48 \text { I } 8 \\
4,3^{2}\end{array}$ & $\begin{array}{c}\text { I IO5 } \\
4401 \\
3,98\end{array}$ & $\begin{array}{l}\text { I } 204 \\
4727 \\
3,9^{2}\end{array}$ & $\begin{array}{c}\text { I I } 88 \\
\\
433^{8} \\
3,64\end{array}$ & $\begin{array}{c}\text { I } 252 \\
4797 \\
3,83\end{array}$ & $\begin{array}{l}\text { I } 220 \\
4410 \\
3,61\end{array}$ \\
\hline
\end{tabular}

\section{RESULTATS}

\section{1. - Croissance.}

\section{a) Rôle de l'antibiotique.}

L'auréomycine atnéliore la croissance d'une façon appréciable. Nous retrouvons une fois de plus un fait bien établi. D'autre part l'érythromycine provoque incontestablement une amélioration de la croissance pondérale du Poulet. Dans tous les cas, les animaux qui reçoivent cet antibiotique sont les plus lourds. En particulier, à dose égale dans la pâtée et dans les mêmes conditions d'élevage, l'érythromycine est toujours plus efficace que l'auréomycine. Nos résultats sont en accord avec ceux de Mc GINNIs, I958 qui reconnaît à l'érythromycine une efficacité supérieure à celle des autres antibiotiques étudiés. 


\section{b) Influence du mode d'élevage.}

A 4 semaines, les lots élevés au sol ont une meilleure croissance que ceux élevés sur grillage. Toutefois cette différence, nette pour les lots " témoins » et "auréomycine ", n'existe pratiquement pas chez les animaux qui reçoivent de l'érythromycine.

Il est loisible de penser que seuls les lots " témoins " et "auréomycine " tirent profit des facteurs de croissance contenus dans la litière, ce qui ne semble pas être le cas des poulets soumis à l'érythromycine. Par contre, à 9 semaines, les résultats se trouvent inversés : dans le cas de régimes identiques, ce sont les poulets élevés sur grillage qui l'emportent en poids sur les animaux élevés au sol.

Dans tous les cas, le rôle favorable joué par l'érythromycine dans la croissance semble peu dépendant des conditions d'élevage. On pourrait interpréter ces faits à la lumière de la théorie qui fait intervenir 1'antibiotique au niveau de la flore microbiemne de l'intestin. I1 se pourrait en effet que, d'une part, 1'érythromycine favorise la synthèse des facteurs de croissance contenus dans les litières par les microbes intestinaux, et que d'autre part, cette synthèse soit plus intense que celle provoquée par l'auréomycine. On pourrait ainsi expliquer la supériorité de l'érythromycine sur l'auréomycine.

c) I’observation des courbes de poids montre que les différences pondérales entre les lots " érythromycine " et "auréonycine " sont les plus importants quand les poussins sont âgés de 4 à 5 semaines. Ce fait est mis en valeur si l'on calcule les augmentations de gain de poids en pourcentage.

\section{TABLFAU II}

\section{Bénéfice pondéral provoqué par les antibiotiques par rapport au témoin}

\begin{tabular}{|c|c|c|c|c|}
\hline & \multicolumn{2}{|c|}{ Élevage sur grillage } & \multicolumn{2}{|c|}{ Elevage au sol } \\
\hline & A 4 semaines & A o semaines & A if scmaines & A 9 semaines \\
\hline $\begin{array}{l}\text { Gain de poids moyen des lots } \\
\text { "Auréomycine" (g) } \ldots . . . . . . . . . \\
\text { Gain de poids des "témoins" (g). }\end{array}$ & $\begin{array}{l}390 \\
367\end{array}$ & $\begin{array}{l}\text { I } 204 \\
\text { I I I } 5\end{array}$ & $\begin{array}{l}404 \\
373\end{array}$ & $\begin{array}{l}1188 \\
\text { I } 105\end{array}$ \\
\hline $\begin{array}{l}\text { Bénéfice pondéral }(g) \ldots \ldots \ldots \ldots \\
\text { Bénéfice en } \% \ldots \ldots \ldots \ldots \ldots\end{array}$ & $\begin{array}{c}23 \\
6,25 \%\end{array}$ & 89 & $8,3^{3 \mathbf{1}}$ & $\begin{array}{r}83 \\
7,5 \%\end{array}$ \\
\hline $\begin{array}{l}\text { Gain de poids moyen des animaux } \\
\text { "Erythronycine" }(g) \ldots \ldots \ldots \ldots, \\
\text { Gain de poids moyen des" témoins" } \\
\text { (g) } \ldots \ldots \ldots \ldots \ldots \ldots \ldots \ldots \ldots \ldots \ldots \ldots\end{array}$ & $\begin{array}{l}433 \\
3^{67}\end{array}$ & $\begin{array}{l}1252 \\
1 \quad 115\end{array}$ & $\begin{array}{l}4.35 \\
27.3\end{array}$ & $\begin{array}{l}1220 \\
1105\end{array}$ \\
\hline $\begin{array}{l}\text { Bénétice pontíral }(g) \ldots \ldots \ldots \ldots \\
\text { Bénéfice en } \because, \ldots \ldots \ldots \ldots \ldots\end{array}$ & $\begin{array}{c}66 \\
\text { I } 8 \%\end{array}$ & $\begin{array}{r}137 \\
\mathrm{I} 2,5 \%\end{array}$ & $\begin{array}{r}62 \\
16,5 \%\end{array}$ & $\begin{array}{r}115 \\
10,3 \%\end{array}$ \\
\hline
\end{tabular}


Au fur et à mesure qu'ils vieillissent, les poulets du lot «auréomycine » ont un gain de poids relatif constant par rapport aux témoins, voisin de 7,5 p. Ioo. Par contre, l'efficacité de l'érythromycine décroît avec l'âge des animaux : 1'avantage observé à 4 semaines est de I 8 p. Ioo sur grillage et de 16,5 p. Ioo au sol; il n'est respectivement que de 12,5 p. Ioo et de Io,3 p. Ioo à 9 semaines. I,e comportement des deux antibiotiques est donc tout différent en fonction de l'âge de l'animal. Ceci est mis en évidence encore mieux dans le tableau III.

TABI TAU III

Bénéfice pondéral provoqué par l'érythromycine par rapport à l'auréomycine

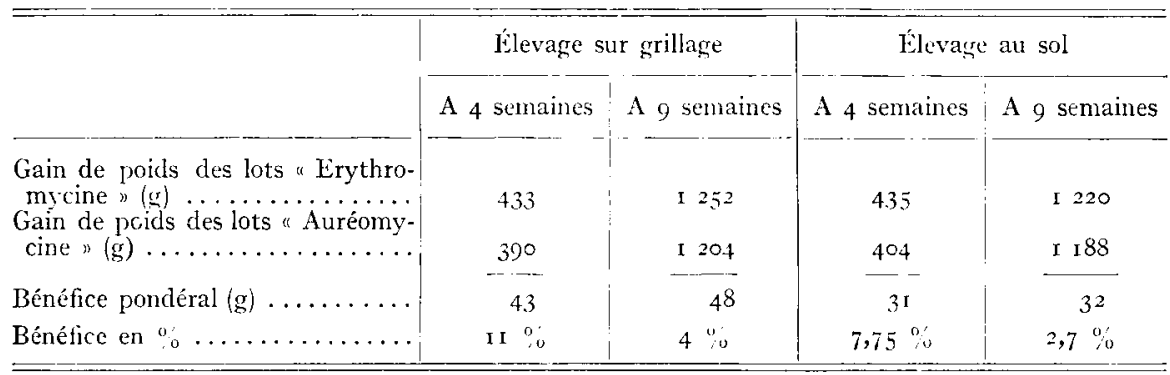

Bien que l'effet positif de l'érythromycine sur la croissance soit spectaculaire pendant les premières semaines de la vie de l'animal — action beaucoup plus marquée que celle de l'auréomycine -, l'avantage du premier antibiotique sur le second est sérieusement compromis lorsque les animaux atteignent le poids commercial où ils seront vendus.

\section{2. - Nourriture ingjérée.}

Les résultats rassemblés dans le tableau I sont les suivants :

A 4 semaines, la quantité de nourriture ingérée est toujours plus forte pour les lots témoins que pour les autres lots. Dans des conditions d'élevage comparables il n'y a pas de différence entre les lots "auréomycine" et "érythromycine".

A 9 semaines et dans des conditions d'élevage identiques les divers régimes ne provoquent aucune différence sur le taux des ingestats. Enfin dans tous les cas, les animaux élevés sur grillage ont consommé plus de nourriture que ceux élevés au sol.

Ces faits se reproduisent de la même manière au niveau de l'indice de consommation. Il semble donc que les animaux élevés au sol tirent un meilleur profit de leurs aliments que ceux élevés sur grillage.

En ce qui regarde l'influence des antibiotiques pour des conditions 
d'élevage semblables, l'indice de consommation est amélioré légèrement par l'auréomycine alors qu'il l'est beaucoup plus dans le cas de l'érythromycine.

\section{3. - Stockage de l'érythromỵcine dans les tissus.}

\section{a) Méthode de dosage.}

Elle est inspirée de celle proposée par Fí́lvier, VACHét et Michel, I955 pour le dosage de l'auréomycine dans les mêmes conditions. Nous utilisons la propriété de l'antibiotique d'inhiber la pousse de Staphylococcus Aureus H ATCC 9r44 entretenu sur un milieu nutritif complet. I'extraction de l'érythromycine est légèrement différente de celle de l'auréomycine. I'extraction de cette dernière prévoit un traitement à $\mathrm{pH}+, 5$ pour précipiter dans l'échantillon la plus grande partie des protéines. I,érythromycine ne supporte pas les milieux acides. Aussi peut-on opérer la séparation des protéines soit par relargage soit par défécation au moyen d'un mélange préalablement neutralisé de sulfate de zinc et de baryte. La première méthode ne nous a pas donné satisfaction car il est toujours difficile d'éliminer totalement le sulfate d'ammoniaque utilisé qui influe ultérieurement sur la croissance du microorganisme servant au dosage. Nous avons préféré la deuxième solution dont les détails opératoires sont indiqués ci-dessous.

\section{b) Extraction.}

L'acétone est un excellent solvant de l'érythromycine. Elle permet de plus une précipitation importante d'une grande partie des protéines. Aussi, ro g de l'échantillon à doser sont broyés finement au mixer pendant Io mn en présence de $50 \mathrm{~cm}^{3}$ d'acétone. Pour éviter que le précipité acétonique n'entraîne par absorption une partie de 1'érythromycine, il est remis continuellement en suspension par agitation. Ia liqueur est alors réfrigérée puis filtrée. I, acétone est évaporée sous vide à $30^{\circ} \mathrm{C}$. On reprend l'érythromycine dans Io $\mathrm{cm}^{3}$ de tampon à $\mathrm{pH} 7$. I a solution ainsi obtenue est trouble et il convient d'effectuer une défécation au moyen du sulfate de zinc, baryte. Ce mélange est préparé au moment de l'emploi à partir d'une solution de baryte à I p. Ioo et d'une solution de sulfate de zinc à $5 \mathrm{p}$. Ioo. On verse cette dernière dans la baryte jusqu'à obtention d'une liqueur neutre. On filtre l'extrait d'érythromycine après précipitation. La solution amenée à un volume connu est prête pour le dosage.

\section{c) Dosage.}

On mesure l'activité inhibitrice de l'extrait sur la pousse de Staphylococcus aureus en la comparant à celle d'une quantité connue d'érythro- 
mycine pure. On opère sur milieu de base liquide ensemencé, possédant la composition suivante:

\begin{tabular}{|c|c|}
\hline xtrait de cour & \\
\hline Autolysat de levure panification .. & $\mathrm{I}, 5$ \\
\hline Peptoine de caséine...$\ldots \ldots \ldots$ & 5 \\
\hline Glucose & I \\
\hline Chlorure de sodium & 3,5 \\
\hline Phosphate bipotassique & 3,68 \\
\hline Phosphate monopotassique & $I, j 2$ \\
\hline Vitrate de potassium & \\
\hline & $\infty$ \\
\hline
\end{tabular}

Le milieu de base est réparti dans des tubes à raison de $9 \mathrm{~cm}^{3}$; la solution d'antibiotique est ajoutée ensuite et 1'on complète à $10 \mathrm{~cm}^{3}$ avec de l'eau distillée.

Lans nos conditions expérimentales, les quantités d'érythromycine apportées dans chaque tube s'échelonnent entre 0 , I et I gamma (o, I à I $\mathrm{cm}^{3}$ d'une solution à t gamma). Chaque tube est reproduit trois fois.

Après une incubation de 3 h 30 au bain-marie à $37^{\circ}$, on arrête la croissance microbienne en ajoutant dans chaque tube une goutte d'une solution au tiers de formol. On apprécie ensuite l'intensité de cette croissance par mesure de la turbidité du liquide au spectrophotomètre de Beckmann B.

\section{d) Résultats.}

A la suite de l'essai de croissance, ro poulets du lot "érythromycine" sont gardés en vue du dosage de cet antibiotique dans les tissus. Ils reçoivent pendant un mois le même régime où la dose d'érythromycine est doublée, soit $40 \mathrm{mg}$ par $\mathrm{kg}$ de régime. Après sacrifice, on prélève le muscle pectoral droit et le foie, qui sont immédiatement broyés dans l'acétone. Les résultats figurent dans le tableau IV.

On observe tout d'abord une grande variabilité des résultats puisque pour un même muscle la quantité d'érythromycine stockée par gramme de tissu passe de 0,20 à 3,02 gamma d'un animal à l'autre. Cette variabilité est toutefois moins importante dans le cas du foie.

Des dosages identiques ont été effectués chez quelques poulets témoins. Voici les résultats.

Le pouvoir antibiotique que l'on retrouve dans les tissus des animaux témoins est négligeable, de l'ordre des erreurs expérimentales. Chez les animaux traités, la teneur des foies en érythromycine est extrêmement faible ( 2 à 3 fois celle des témoins). Les muscles manifestent une aptitude plus marquée à retenir l'antibiotique, qui se traduit par un stockage représentant un taux variant de I à I 5 fois la valeur trouvée chez les témoins. Bien que l'on ne puisse pas interpréter cette variabilité énorme, il demeure cependant, qu'en valeur absolue, les quantités d'érythromycine retrouvées sont minimes et ne peuvent pas représenter de danger pour le consomma- 
'I'ABLEAU IT

Stockage de l'érythromycine dans les tissus

\begin{tabular}{c|c|c}
\hline $\begin{array}{c}\text { Numéro } \\
\text { des poulets }\end{array}$ & Foic $\gamma / \mathrm{g}$ & Muscle $\gamma / \mathrm{g}$ \\
\hline $\mathrm{I}$ & 0,5, & 0,20 \\
\hline 2 & 0,50 & 0.20 \\
\hline 3 & 0,32 & $\mathrm{I}, 2 \mathrm{I}$ \\
\hline 4 & 0,90 & 2,90 \\
\hline 5 & 0,84 & 3,02 \\
\hline 6 & 0,55 & 0,50 \\
\hline 7 & 0,66 & 0,90 \\
\hline 8 & 0,85 & 1,40 \\
\hline 9 & 0,97 & 1,50 \\
\hline 10 & 0,40 & 2,90 \\
\hline
\end{tabular}

TABLEAT $Y$

Activité antibiotique des tissus des poulets témoins

\begin{tabular}{c|c|c}
\hline $\begin{array}{c}\text { Numéro } \\
\text { des poulets }\end{array}$ & Foie $\gamma / \mathrm{g}$ & Muscle $\mathrm{r} / \mathrm{g}$ \\
\hline $2 \mathrm{r}$ & 0,23 & 0,27 \\
\hline 22 & 0 & 0,15 \\
\hline
\end{tabular}

teur. Nos résultats sont en accord avec ceux de CHENG-CHUx I EE et al., I953, qui ne retrouvent pratiquement pas d'érythromycine dans le muscle du Rat $7 \mathrm{~h}$ après une administration orale d'une dose unique de $20 \mathrm{mg}$ de l'antibiotique.

\section{CONCLUSION}

Mieux que l'auréomycine, l'érythromycine pure améliore la croissance pondérale et l'indice de consommation du Poulet. Ce nouvel antibiotique employé dans l'alimentation des jeunes volailles provoque une amélioration très importante de la croissance du Poussin. Le bénéfice peut atteindre la valeur de $I 8$ p. Ioo. Ce résultat est encore plus démonstratif si l'on compare l'effet de croissance de l'érythromycine à celui d'un autre 
antibiotique, l'auréomycine. A l'àge de quatre semaines l'augmentation du gain de poids est deux fois plus élevée quand les aninaux reçoivent de l'érythromycine que lorsqu'ils sont traités à l'auréomycine dans les mêmes conditions. 'Toutefois, sous l'effet de l'érythromycine, le bénéfice pondéral va en s'amenuisant au fur et à mesure que l'animal devient plus âgé, et à 9 semaines, l'amélioration du taux de croissance dù à cet antibiotique -est de l'ordre de celle obtenue avec l'auríonycine.

Le dépôt d'érythromycine dans le foic et danss le muscle est extrêmement faible.

\section{SUMMARY}

Erythromycine added to a commercial diet for chicks at a dose of $20 \mathrm{~g}$ per ton of feed produces an improvement in growth and feed 'efficiency which is superior to that obtained by aureomycine used in the same conditions. For chicks of 4 weeks of age, erythromicine gives a gain in weight of 18 p. Ioo, and aureomycine $8 \mathrm{p}$. Ioo. However, as the birds grow older, the efficiency of the two drugs tends to become similar.

The weight increase produced by erythromycine remains the same when the chicks are raised on "shavings litter " or on " raised wire floor".

\section{REFERENCLS BIBLIOGRAPHIQUES}

Chen-Chun Lee, Axperson R. C., Chen K. K., I953. 'Tissue distribution of Erythromycine in rat. Antibiot. and Chemothcrapy, U. S. A., 3, 920924.

Février R., VAchei, J. P., Michil M. C., I955. Les antibiotiques dans l'alimentation du porc. III. Stockage des antibiotiques dans les organes et les tissus. Ann. Zootech., IV, I39-I43.

Fraxçors A. C., Micher M. C., I955. Action de la pénicilline et de l'auréomycine sur les propriétés désaminantes de la flore intestinale du porc. C. R. Acad. Sci., 240, I24-126.

HUNTER 'T. H., I950. Speculations on the mechanism of cure of bacterial endocarditis. J. Amer. Med. Assoc., 144, 524-527.

MC Ginnis J., MerRili, L. H., FRY R. E., I958. Use-History of antibiotics as related to their efficacy in promoting growth of turkeys. Poult. Sci., 37, 8I0-8I3.

Michei, M. C., Françors A. C., I955. Relation entre l'influence des antibiotiques sur la croissance du porc et l'inhibition des désaminases de la flore intestinale. C. R. Acad. Sci., 240, 808-8Io.

Michei, M. C., I958. Métabolisme de la flore intestinale du porc. Tiré des travaux de la Commission des antibiotiques du C. N. E. R. N. A. Ann. Nutr. Alim., 12, I52-I6r.

Rerat A., Abraham J., Champigny O., Jacquot R., I957. Etude comparée de l'érythromycine et de la levure sur la croissance et la rétention azotée du Rat blanc. C. R. Acad. Sci., 245, 2I3-216.

RERAT A., Escribano L., JacQuot R., I957. Effets comparés de deux nouveaux antibiotiques, l'éythromycine et la soframycine sur la croissance pondérale du rat et considérations sur le mécanisme d'action de 1'antibiosupplémentation. Arch. Sci. Physiol., 11, 331-346. 